\title{
Evaluation of Cane Genotypes Under Sprinkler Irrigation at Early Selection Stage for Tolerance to Sugarcane Streak Mosaic Virus (SCSMV) at Ferké Sugar Estates in Ivory Coast
}

\author{
Crépin B. PÉNÉ ${ }^{1} \&$ Yavo Y.M. BÉHOU ${ }^{2}$ \\ ${ }^{1}$ SUCAF-CI/SOMDIAA Group, Research \& Development Department, Ivory Coast \\ ${ }^{2}$ EDP/INP-HB, Department of Agronomy, Agricultural Engineering and Forestry, Yamoussoukro, Ivory Coast \\ Correspondence: Crépin B. PÉNÉ, SUCAF-CI/SOMDIAA Group, Research \& Development Department, 22 rue \\ des Carrossiers, Treichville Zone 3, P.O. Box 1967 Abidjan 01, Ivory Coast. Tel: 225-4783-1916. Email: \\ bpene@sucafci.somdiaa.com/cbpene20@yahoo.com
}

Received: April 3, 2020 Accepted: April 10, 2020 Online Published: April 16, 2020

\begin{abstract}
Sugarcane streak mosaic virus (SCSMV) became the major endemic disease of economic importance in Ivorian sugar estates almost two years ago, which spreads very fast across plantations and varieties. The study aimed to determine resistant sugarcane genotypes against SCSMV in Ferké sugar estates. It involved five experiments conducted at first selection stage under sprinkler irrigation, following a randomized complete block design (RCBD) with 20 to 30 different genotypes, two check varieties included, all in 4 replicates. Experiments were planted in October or December 2018, and expected to be harvested in November/December 2019 and 2020 as plant cane and first ratoon, respectively. Disease incidence and severity across all experiments were determined at 3 to 4 months, i.e. at early formative growth stage where symptoms due to SCSMV could be easily observed and recognized in the field. In each of the five selection trials conducted on both Ferké $1 \& 2$ sugar estates, highly significant differences in disease incidence and severity were observed between genotypes as well as crop cycles (plant cane and first ratoon). Except for one trial (B3-24 in Ferké 1), Genotype x crop cycle interactions were significant or highly significant, which showed that the majority of genotypes tested behaved differently from plant cane to first ratoon towards the disease. Particularly, the percentage of resistant genotypes decreased from 50 to $3.4 \%$ whereas that of highly susceptible ones increased from 4.2 to $92.4 \%$. This shows the level of challenge to be tackled in the control of SCSM disease through sugarcane breeding and selection. At the end of the current selection stage under way, i.e. after harvest of first ratoon, only the best yielding genotypes among the resistant ones will undergo the advanced selection stage.
\end{abstract}

Keywords: natural infection, disease susceptibility, varietal resistance, growth stage, insect vector, yield loss, disease epidemiology.

\section{Introduction}

Sugarcane is one of the important cash crops grown in Ivory Coast, covering approximately 35,500 ha of land surface with an average yield of 70-75 t of cane/ha irrigated and rainfed crops combined. Sugarcane is mostly cultivated under irrigated conditions contributing to around $90 \%$ of Ivorian sugar production, i.e. about 200,000 $\mathrm{t} /$ year provided from four different factories.

Sugarcane streak mosaic (SCSM) is a newly emerging disease in the country, which has been infecting that crop since 2017. The virus was known about fifteen years ago in Asian and Latino American sugarcane producing countries where it was first reported in 2004 and 2005 respectively in Brazil (Gonçalves et al, 2004; Gonçalves et al, 2007a; Gonçalves et al, 2007b; Gonçalves et al, 2011; Gonçalves et al, 2012) and Java, Indonesia (Chatenet et al, 2005; Kristini et al, 2006). It was reported that at least two strains of SCSMV were infecting sugarcane in Asia (Putra et al, 2015a). SCSMV is a Poacevirus from the Potyviridae family, which is easily transmitted through plant extracts and vertically through sugarcane cuttings. It infects a limited species of Poaceae family such as sugarcane, sorghum, maize, Dacttyloctenium aegyptium, Pennisetum glaucum, Digitaria delilis as reported by several investigators (Hema et al, 2001; Xu et al, 2010; Putra \& Damayanti, 2012; Putra et al, 2014). However, no insect vector of the pathogen was reported so far (Putra et al, 2015a, Addy et al, 2017). Several species of aphids have been tested including Aphis cracivora, Rhopalosiphum maidis and Ceratovacuna lanigera and it was reported that 
they could not transmit the virus (Hema et al, 2001; Putra \& Damayanti, 2012; Putra et al, 2014). It was reported that SCSMV could be detected using RT-PCR on sugarcane aphid colony (Melanaphis indosacchari), and still the mechanism of virus transmission needs to be studied so as to develop an integrated pest management strategy (Brown, 1997).

Across Ivorian sugar estates, except for a limited number of varieties like M2593/92, M1400/86, SP70-1143, R584 and R91-2021, all main cultivars were found moderately or highly susceptible to the disease. It is the case of varieties like FR80-69, R579, SP70-1006, SP71-1406, SP71-8210, SP81-3250, R573, R570, Co997 and R575. Cane and sugar yield losses due to SCSMV occurred in Zuénoula sugar estate in central Ivory Coast where the disease broke out in 2017 on variety R575, were estimated to 20 to $30 \%$ over the last two cropping seasons (201718 and 2018-19). The disease expansion was so fast in Zuénoula plantations that $20 \%$ of land under cultivation with moderately or highly susceptible varieties were replanted every year instead of $10 \%$ as usual (Béhou \& Péné, 2019). The disease broke out in Ferké sugar estates of northern Ivory Coast in June 2018 on variety FR80-69, a highly susceptible one, and within 12 months, it has spread to all major varieties cultivated like R579, SP71-1406, SP70-1006 and SP71-8210, with a severity score of 2 or 3, 4 being the highest symptom level. Total cropped land concerned was estimated to 2000 ha, i.e. about $25 \%$. The threat on sugar production was so crucial that a replantation strategy was planned to be implemented over the next three or four years.

The study aimed to determine under irrigation resistant sugarcane genotypes of Reunion and Ivorian origin against SCSMV.

\section{Materials and Methods}

\subsection{Site Characteristics}

The study was carried out on four sugarcane plantations (B3-13, B3-24, V4-43, V8-01) and an experimental station (P3-61), at Ferké 1 and Ferké 2 sugar estates, in northern Ivory Coast $\left(9^{\circ} 20^{\prime}-9^{\circ} 60^{\prime} \mathrm{N}, 5^{\circ} 22^{\prime}-5^{\circ} 40^{\prime} \mathrm{O}, 325 \mathrm{~m}\right.$ a.s.l.). The prevailing climate is tropical dry with two seasons: one, from November to April, is dry and the other, from May to October, is wet. The dry season is marked by the northern trade wind, which blows over midNovember to late January. The rainfall pattern is unimodal with a focus on August and September which total amount of rainfall reaches almost half of the average annual rainfall $(1200 \mathrm{~mm})$ with an average daily temperature of $27{ }^{\circ} \mathrm{C}$. Average maximum and minimum daily air temperatures are 32.5 and $21{ }^{\circ} \mathrm{C}$, respectively. To meet sugarcane crop water requirements, the total amount of irrigation water required reaches $700 \mathrm{~mm} /$ year (Konan et al, 2017a,b). Both Ferké sugar mill plantations cover around 15500 ha with 10000 ha under irrigation and 3500 ha of rainfed village plantations, lie mainly on shallow or moderately deep soils built up on granites. Main soil units encountered are ferralsols and temporally waterlogged soils in valley bottoms of Bandama and Lokpoho river basins with a sandy-clay texture.

\subsection{Experimental Design}

All experiments were carried out over 12 months as plant cane following a randomized complete block design (RCBD) with 20 to 30 cane genotypes and two commercial varieties as control (M2593/92 and R579), in 3 replicates. Each sugar estate was equipped with a weather station where parameters required to determine crop $\mathrm{ET}_{0}$ like solar radiation, average daily air temperature, relative air moisture, and wind speed were measured. Rainfall data were recorded from different rain gauges L1-105, but also P3-61, V4-15 and V8-32 located close to Ferké 1 and Ferké 2 experiments, respectively. Ferké 1 experiments were planted on October 25 and December 11, 2018 were expected to be harvested 11 or 12 months later, i.e. in November or December 2019 and 2020, respectively, as plant cane and first ratoon. Those of Ferké 2 were planted on October 12, December 20 and 29, 2018 and expected to be harvested in November or December 2019 and 2020, respectively. Each plot was composed of 5 dual rows of $5 \mathrm{~m}$ long with $0.50 \mathrm{~m}$ and $1.90 \mathrm{~m}$ of row spacings. Field management in terms of fertilizer and herbicide applications were done according to usual practices in commercial plantations. NKP fertilizer (16-8.5-23\%) was applied mechanically at the routine rates of $500 \mathrm{~kg} / \mathrm{ha}$ in rainfed plant cane. Preemergence chemical weeding based on pendimethalin combined with clorimuron-ethyl (3.5 1/ha) was achieved mechanically two days after planting.

\subsection{Genotype Infections Investigated}

Three to four months after planting, all genotypes being tested were observed for symptom detection of SCMV (sugarcane streak mosaic Virus) under natural conditions. Ratings recorded were based on symptoms observed on sugarcane leaves. Four different levels of SCSM disease symptoms were as follows: (1) mild streak, (2) moderately streak, (3) high streak and (4) very high streak. 

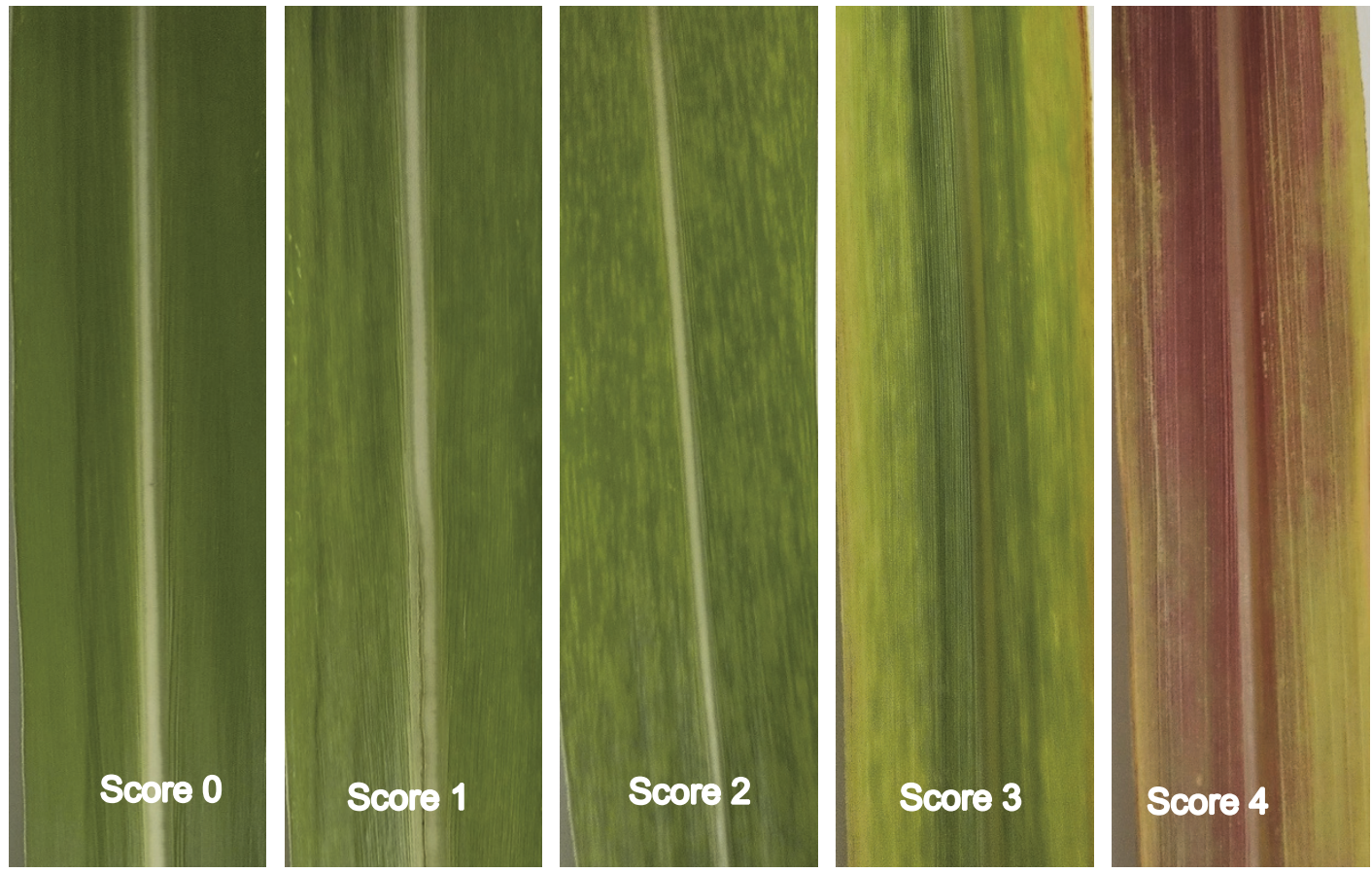

Figure 1. Severity scale of SCSMV infection in sugarcane. Leaf 0 is asymptomatic and Leaf 4 very highly infected. Variety R579 leaves collected in Ferké. Scale adapted from Putra et al (2014).

Disease incidence (Inc) is defined as the percentage of infected tillers, no matter the severity, over the total number of tillers observed within two central micro-plots made of one dual row of $5 \mathrm{~m}$ long with respect to each genotype and replication.

$$
\text { Inc }(\%)=(\mathrm{Nb} \text { of Infected tillers / Total } \mathrm{Nb} \text { of tillers }) \times 100
$$

Disease incidence was classified as highly resistant or asymptomatic $(0 \%)$, moderately resistant $(<10 \%)$, susceptible ([10-40\%[) and highly susceptible ( $\geq 40 \%)$.

Disease severity (Sev) is defined as the average score or rating of infected tillers with respect to their severity over the total number of tillers observed within two central micro-plots made of one dual row of $5 \mathrm{~m}$ long $\left(9.5 \mathrm{~m}^{2}\right)$ with respect to each genotype and replication.

$$
\begin{aligned}
& \operatorname{Sev}(-)=\left(\mathrm{N}_{0} \mathrm{x} 0+\mathrm{N}_{1} \mathrm{x} 1+\mathrm{N}_{2} \times 2+\mathrm{N}_{3} \times 3+\mathrm{N}_{4} \times 4\right) /\left(\mathrm{N}_{0}+\mathrm{N}_{1}+\mathrm{N}_{2}+\mathrm{N}_{3}+\mathrm{N}_{4}\right)=\left(\mathrm{N}_{1} \times 1+\mathrm{N}_{2} \times 2+\mathrm{N}_{3} \times 3+\right. \\
& \left.\mathrm{N}_{4} \times 4\right) /\left(\mathrm{N}_{0}+\mathrm{N}_{1}+\mathrm{N}_{2}+\mathrm{N}_{3}+\mathrm{N}_{4}\right)
\end{aligned}
$$

Where $\mathrm{N}_{0}$ : $\mathrm{Nb}$ of asymptomatic tillers; $\mathrm{N}_{1}: \mathrm{Nb}$ of mild streak tillers, $\mathrm{N}_{2}$ : Nb of moderately streak tillers, $\mathrm{N}_{3}: \mathrm{Nb}$ of higly streak tillers, $\mathrm{N}_{4}$ : $\mathrm{Nb}$ of very highly streak tillers.

\subsection{Statistical Analyses}

The quantitative data recorded in this study were subjected to the analysis of variance, using statistical procedures described by Gomez and Gomez (1984) and reported by Shitahum et al (2018) with the assistance of R software package version 3.5.2. Differences between means of treatments were determined from HSD's test.

\section{Results and Discussion}

\subsection{Climatic Conditions Over Plant Cane and First Ratoon Crop}

As expected, both experimental sites presented a similar rainfall patterns with a per-humid season taking place from June to October (Fig 1). The dry season which took place from November 2018 to May 2019, and from October 2019 to February 2020, was marked by irrigation applications.

In Ferké 1 experiments, total rainfall and reference evapotranspiration (ETo) recorded across crop cycle gave $1538.5 \mathrm{~mm}$ on average regarding two locations and $2188 \mathrm{~mm}$, respectively. Total rainfall deficit over crop growing season from October 2018 to February 2020 gave $895-961 \mathrm{~mm}$ and the average daily temperature across crop cycle varied from 24.4 to $34.5^{\circ} \mathrm{C}$. 
In Ferké 2 experiments, total precipitation and reference evapotranspiration (ETo) recorded across crop cycle gave $1656 \mathrm{~mm}$ on average regarding three locations and $2075 \mathrm{~mm}$, respectively. Total rainfall deficit obtained over crop growing season from October 2018 to February 2020 gave $904-958 \mathrm{~mm}$ whereas the average daily temperature across crop cycle varied from 24.7 to $30.5^{\circ} \mathrm{C}$.
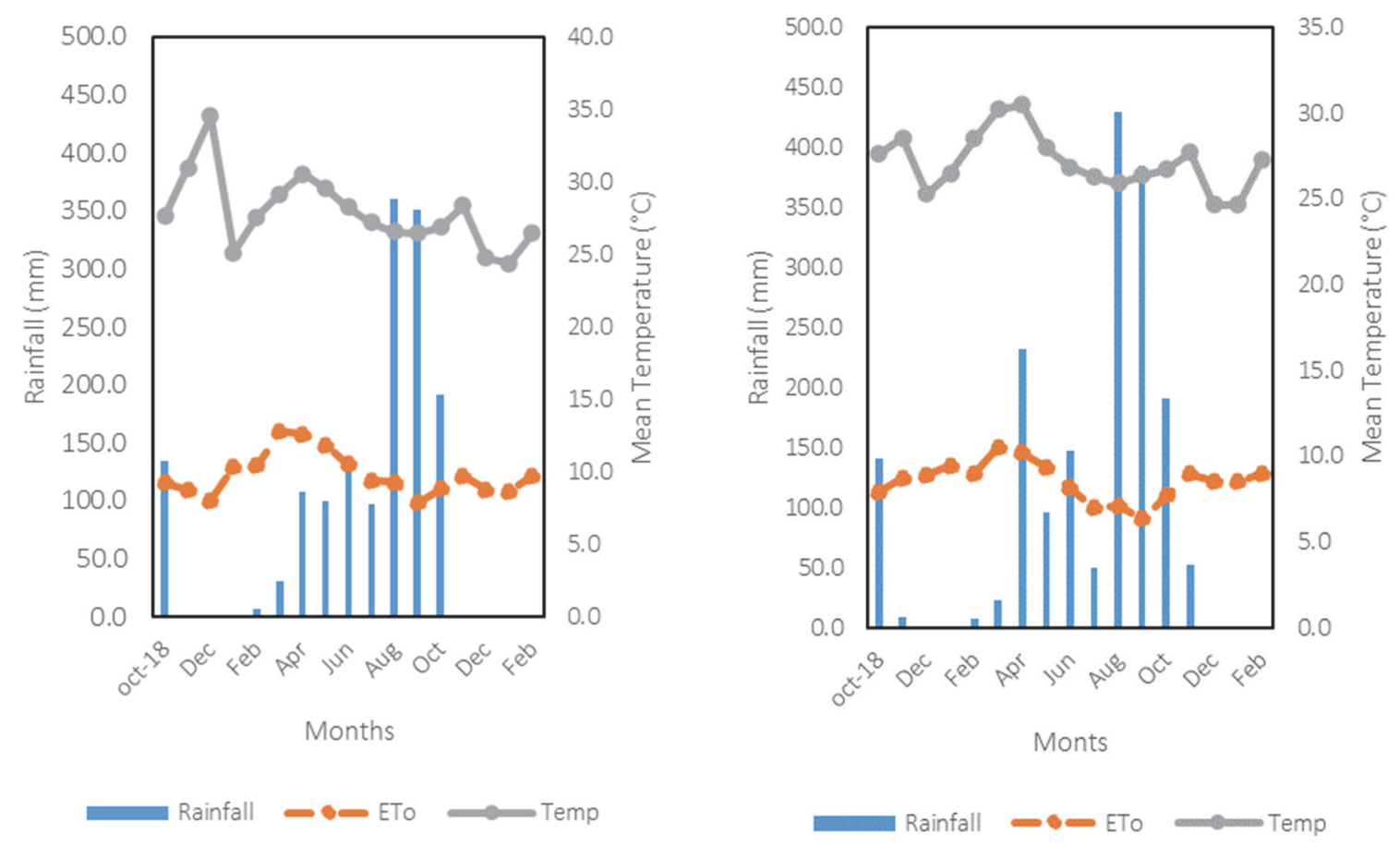

Figure 1a. Climate on Ferké 1 experimental sites (B3-

Figure 1b. Climate on Ferké 2 experimental site of P3-61. $13 / 24)$.
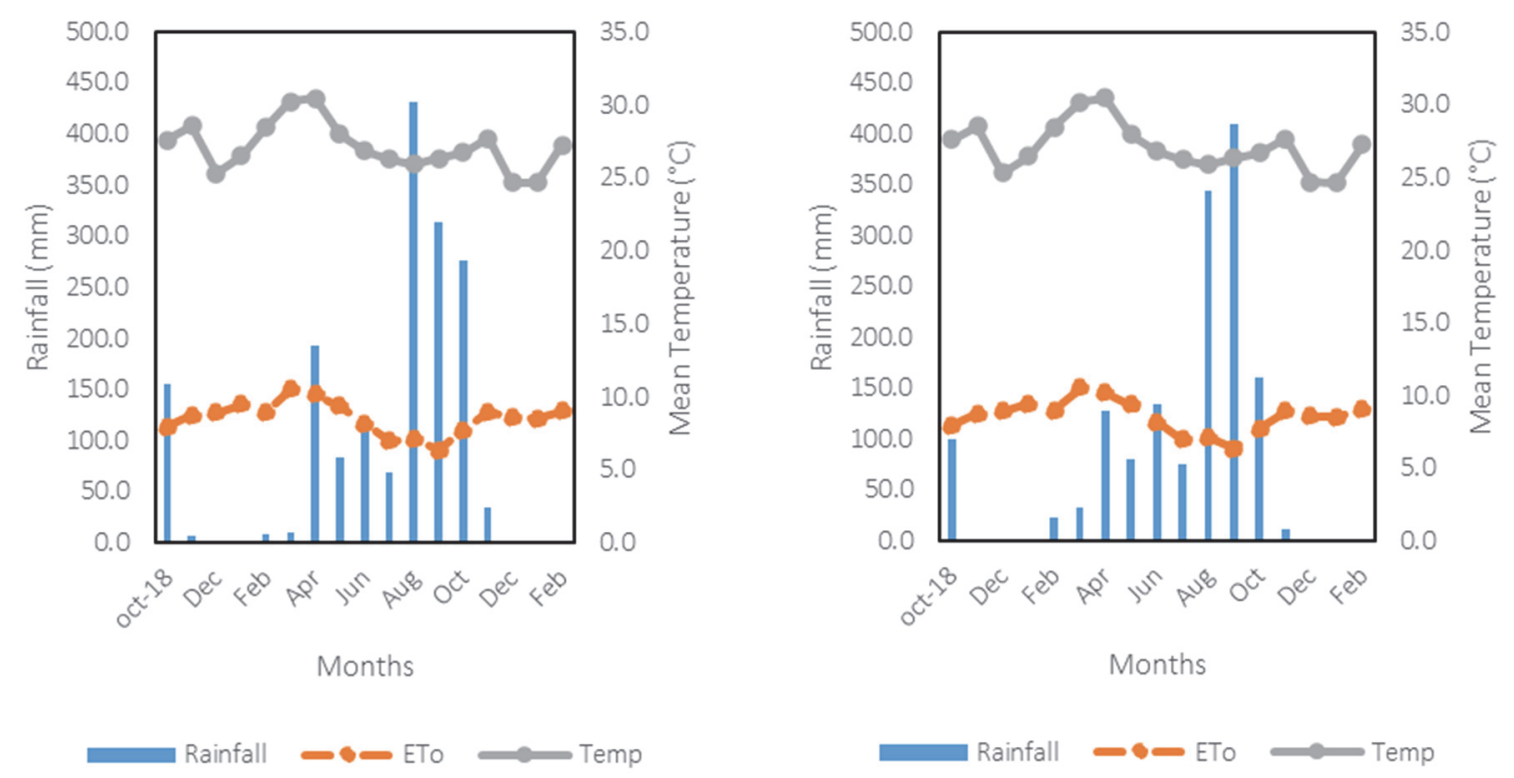

Figure 1c. Climate on Ferké 2 experimental site of V4-43. Figure 1d. Climate on Ferké 2 experimental site of V8-01 Figure 1. Prevailing climate on Ferké 1 and Ferké 2 experimental sites in plant cane and first ratoon crop, Ivory Coast 


\subsection{Cane Genotypes as Affected by SCSM Disease in FerkÉ 1 Experiments}

In both Ferké 1 trails (B3-13 and B3-24 field plots), highly significant differences $(\mathrm{P}<0.1)$ in disease incidence and severity were observed between genotypes as well as crop cycles (Table 1). Genotype $\mathrm{x}$ crop cycle interactions were significant or highly significant in B3-13 trial, while not significant in B3-24 trial. This shows that in B3-13 trial, cane genotypes behaved differently from plant cane to first ratoon towards the disease. Particularly, many asymptomatic genotypes assumed to be tolerant or resistant in plant cane became susceptible in first ratoon. In contrast, for the B3-24 trial, genotypes tested behaved similarly over both crop cycles in terms of their ranking, although the disease symptoms worsened sometimes in first ratoon.

High values of coefficient of variation determined in both experiments $(70-87 \%)$ could be explained by natural infection of disease, although that one became endemic over the last two years in Ferké sugar estates. Similar values were reported in survey on major endemic diseases conducted in the same agro-ecology (Béhou \& Péné, 2018; 2019).

Table 1. SCSM disease incidence and severity on cane genotypes at 3 months in plant cane and first ratoon regarding Ferké 1 experiments, Ivory Coast

\begin{tabular}{|c|c|c|c|c|c|}
\hline \multicolumn{3}{|c|}{ Ferké 1 Trial B3-13 } & \multicolumn{3}{|c|}{ Ferké 1 Trail B3-24 } \\
\hline Genotypes & $\%$ Incidence & Severity & Genotypes & $\%$ Incidence & Severity \\
\hline M2593-92 & $36.3 \mathrm{bc}$ & $0.5 \mathrm{bc}$ & M2593/92 & $31.9 \mathrm{~cd}$ & $0.3 \mathrm{~cd}$ \\
\hline RCI10/164 & $26.3 \mathrm{de}$ & $0.3 \mathrm{ef}$ & RCI11/1110 & $35.1 b c$ & $0.4 \mathrm{bc}$ \\
\hline RCI11/163 & $47.8 \mathrm{bc}$ & $0.5 b c$ & RCI11/1111 & $43.7 \mathrm{ab}$ & $0.5 \mathrm{ab}$ \\
\hline RCI11/167 & $41.5 b c$ & $0.4 \mathrm{~cd}$ & RCI11/1112 & $44.8 \mathrm{ab}$ & $0.4 \mathrm{ab}$ \\
\hline RCI11/168 & $41.4 \mathrm{bc}$ & $0.4 \mathrm{bc}$ & RCI11/1113 & $49.6 \mathrm{ab}$ & $0.5 \mathrm{ab}$ \\
\hline RCI11/169 & $40.8 b c$ & $0.4 \mathrm{bc}$ & RCI11/1114 & $47.8 \mathrm{ab}$ & $0.5 \mathrm{ab}$ \\
\hline RCI11/170 & $24.4 \mathrm{ed}$ & $0.2 \mathrm{fg}$ & RCI12/1115 & 25.0de & $0.3 \mathrm{ef}$ \\
\hline RCI13/172 & $55.7 \mathrm{ab}$ & $0.6 b c$ & RCI12/1116 & $27.5 \mathrm{de}$ & $0.3 \mathrm{de}$ \\
\hline RCI13/175 & $54.3 \mathrm{ab}$ & $0.6 \mathrm{bc}$ & RCI13/1117 & $31.6 \mathrm{~cd}$ & $0.3 \mathrm{~cd}$ \\
\hline RCI13/176 & 40.3 & $0.4 \mathrm{~cd}$ & RCI13/1118 & $55.9 \mathrm{ab}$ & $0.6 \mathrm{ab}$ \\
\hline RCI13/178 & $75.4 \mathrm{a}$ & $0.9 \mathrm{a}$ & RCI13/1119 & $39.4 b c$ & $0.4 \mathrm{ab}$ \\
\hline RCI13/181 & 30.9 & $0.3 \mathrm{de}$ & RCI13/1120 & $40.5 \mathrm{ab}$ & $0.4 \mathrm{ab}$ \\
\hline RCI13/182 & $42.5 \mathrm{ab}$ & $0.7 \mathrm{ab}$ & RCI13/1121 & $22.0 \mathrm{f}$ & $0.2 \mathrm{f}$ \\
\hline RCI13/183 & 19.3 & $0.2 \mathrm{~g}$ & RCI13/1122 & $36.4 b c$ & $0.4 \mathrm{bc}$ \\
\hline RCI13/184 & 32.8 & $0.3 \mathrm{de}$ & RCI13/1123 & $41.5 \mathrm{ab}$ & $0.4 \mathrm{ab}$ \\
\hline RCI13/185 & 44.9 & $0.5 b c$ & RCI13/1124 & $30.3 \mathrm{~cd}$ & $0.3 \mathrm{~cd}$ \\
\hline RCI13/186 & $58.3 \mathrm{ab}$ & $0.6 b c$ & RCI13/197 & $22.6 \mathrm{ef}$ & $0.2 \mathrm{f}$ \\
\hline RCI14/158 & 38.4 & $0.4 \mathrm{bc}$ & RCI13/198 & $52.4 \mathrm{ab}$ & $0.6 \mathrm{ab}$ \\
\hline RCI14/160 & $56.1 \mathrm{ab}$ & $0.6 b c$ & RCI14/1100 & $52.2 \mathrm{ab}$ & $0.5 \mathrm{ab}$ \\
\hline \multirow[t]{11}{*}{ RCI14/161 } & 38.8 & $0.4 \mathrm{~cd}$ & RCI14/1101 & $44.5 \mathrm{ab}$ & $0.5 \mathrm{ab}$ \\
\hline & & & RCI14/1102 & $52.1 \mathrm{ab}$ & $0.6 \mathrm{ab}$ \\
\hline & & & RCI14/1103 & $61.0 \mathrm{ab}$ & $0.6 \mathrm{ab}$ \\
\hline & & & RCI14/1104 & $52.4 \mathrm{ab}$ & $0.6 \mathrm{ab}$ \\
\hline & & & RCI14/1105 & $49.0 \mathrm{ab}$ & $0.5 \mathrm{ab}$ \\
\hline & & & RCI14/1106 & $57.7 \mathrm{ab}$ & $0.6 \mathrm{ab}$ \\
\hline & & & RCI14/1107 & $47.4 \mathrm{ab}$ & $0.5 \mathrm{ab}$ \\
\hline & & & RCI14/1108 & $45.5 \mathrm{ab}$ & $0.5 \mathrm{ab}$ \\
\hline & & & RCI14/1109 & $43.5 \mathrm{ab}$ & $0.4 \mathrm{ab}$ \\
\hline & & & RCI14/1125 & $67.2 \mathrm{a}$ & $0.7 \mathrm{a}$ \\
\hline & & & RCI14/199 & $39.0 \mathrm{bc}$ & $0.4 \mathrm{ab}$ \\
\hline Replications & $* *$ & $* *$ & Replications & Ns & Ns \\
\hline Genotypes (G) & $* * *$ & $* * *$ & Genotypes (G) & $* *$ & $* *$ \\
\hline Crop cycle (Y) & $* * *$ & $* * *$ & Crop cycle (Y) & $* * *$ & $* * *$ \\
\hline Gx Y & $*$ & $* *$ & Gx Y & Ns & Ns \\
\hline Mean & 43.3 & 0.5 & Mean & 43.0 & 0.4 \\
\hline CV (\%) & 74.8 & 70.1 & CV (\%) & 86.8 & 86.4 \\
\hline SD & 32.4 & 0.3 & $\mathrm{SD}$ & 37.3 & 0.3 \\
\hline
\end{tabular}




\subsection{Cane Genotypes as Affected by SCSM Disease at Three Months in Ferké 2 Experiments}

In the three trails of Ferké 2 location (P3-61, V4-43 and V8-01 field plots), highly significant differences ( $\mathrm{P}<0.1)$ in disease incidence and severity were observed between genotypes as well as crop cycles (Table 2). Genotype $x$ crop cycle interactions were significant in the P3-61 trial and highly significant in V4-43 and V8-01 trails. This corroborates the fact the majority of genotypes tested behaved differently from plant cane to first ratoon towards the disease. Particularly, many asymptomatic or moderately resistant genotypes in plant cane became susceptible or highly susceptible in first ratoon. Similarly to both Ferké 1 trails, high values of coefficient of variation determined in both experiments (78.5 - 101\%) could be explained by natural infection of disease.

Table 2. SCSM disease incidence and severity on cane genotypes in plant cane and first ratoon at 3 months regarding Ferké 2 experiments, Ivory Coast

\begin{tabular}{|c|c|c|c|c|c|c|c|c|}
\hline \multicolumn{3}{|c|}{ Ferké 2 Trial P3-61 } & \multicolumn{3}{|c|}{ Ferké 2 Trial V4-43 } & \multicolumn{3}{|c|}{ Ferké 2 Trial V8-01 } \\
\hline Genotypes & Incid.(\%) & Severity & Genotypes & Incid.(\%) & Severity & Genotypes & Incid.(\%) & Severity \\
\hline M2593/92 & $20.4 \mathrm{~cd}$ & 0.2 & M2593/92 & 50.3 & 0.8 & $\mathrm{R} 579$ & 53.5 & 0.7 \\
\hline RCI12/149 & 42.2 & 0.4 & RCI11/112 & 32.5 & 0.3 & RCI11/1128 & 59.4 & 0.7 \\
\hline RCI13/138 & 38.3 & 0.5 & RCI11/134 & 38.1 & 0.5 & RCI11/1129 & 55.4 & 0.6 \\
\hline RCI13/140 & 46.4 & 0.5 & RCI11/135 & 26.1 & 0.3 & RCI11/1146 & 58.9 & 0.6 \\
\hline RCI13/141 & 50.3 & 0.6 & RCI11/162 & 45.0 & 0.5 & RCI12/1130 & 60.8 & 1.0 \\
\hline RCI13/142 & 31.9 & 0.3 & RCI11/165 & 58.5 & 0.7 & RCI13/1131 & 61.6 & 0.7 \\
\hline RCI13/143 & 49.5 & 0.6 & RCI11/166 & 54.6 & 0.7 & RCI13/1132 & 2.3 & 0.0 \\
\hline RCI13/144 & 38.1 & 0.5 & RCI11/190 & 57.2 & 0.8 & RCI13/1133 & 72.9 & 0.8 \\
\hline RCI13/145 & 1.4 & 0.0 & RCI12/191 & 47.1 & 0.6 & RCI13/1134 & 54.5 & 0.6 \\
\hline RCI13/150 & 33.7 & 0.3 & RCI12/192 & 12.6 & 0.1 & RCI13/1135 & 70.6 & 0.8 \\
\hline RCI13/151 & 39.3 & 0.4 & RCI13/110 & 33.6 & 0.4 & RCI13/1136 & 40.2 & 0.4 \\
\hline RCI13/152 & 45.0 & 0.5 & RCI13/13 & 37.4 & 0.4 & RCI13/1148 & 58.7 & 0.8 \\
\hline RCI13/153 & 26.3 & 0.3 & RCI13/139 & 32.0 & 0.3 & RCI14/1126 & 66.5 & 0.9 \\
\hline RCI14/146 & 50.1 & 0.6 & RCI13/16 & 3.5 & 0.0 & RCI14/1127 & 55.2 & 0.7 \\
\hline RCI14/147 & 42.0 & 0.5 & RCI13/173 & 51.0 & 0.8 & RCI14/1137 & 53.3 & 0.7 \\
\hline RCI14/148 & 54.2 & 0.6 & RCI13/174 & 54.6 & 0.9 & RCI14/1138 & 53.5 & 0.9 \\
\hline RCI14/154 & 39.0 & 0.4 & RCI13/177 & 64.6 & 1.1 & RCI14/1139 & 48.1 & 0.7 \\
\hline RCI14/155 & 48.2 & 0.8 & RCI13/179 & 66.6 & 0.8 & RCI14/1140 & 49.2 & 0.5 \\
\hline RCI14/156 & 44.3 & 0.5 & RCI13/180 & 59.8 & 1.0 & RCI14/1141 & 48.6 & 0.5 \\
\hline \multirow[t]{11}{*}{ RCI14/157 } & 29.2 & 0.3 & RCI13/187 & 2.2 & 0.0 & RCI14/1142 & 52.1 & 0.7 \\
\hline & & & RCI13/193 & 35.4 & 0.4 & RCI14/1143 & 57.3 & 0.8 \\
\hline & & & RCI13/194 & 17.3 & 0.2 & RCI14/1144 & 57.2 & 0.9 \\
\hline & & & RCI13/195 & 48.2 & 0.6 & RCI14/1145 & 36.6 & 0.4 \\
\hline & & & RCI13/196 & 43.5 & 0.5 & RCI14/1147 & 52.5 & 0.7 \\
\hline & & & RCI14/111 & 36.3 & 0.4 & & & \\
\hline & & & RCI14/128 & 35.4 & 0.3 & & & \\
\hline & & & RCI14/159 & 24.5 & 0.3 & & & \\
\hline & & & RCI14/171 & 55.8 & 0.6 & & & \\
\hline & & & RCI14/188 & 47.7 & 0.5 & & & \\
\hline & & & RCI14/189 & 51.2 & 0.8 & & & \\
\hline Replications & $* * *$ & $* * *$ & Replications & Ns & Ns & Replications & Ns & Ns \\
\hline Genotyp (G) & $* * *$ & $* * *$ & Genotyp (G) & $* * *$ & $* * *$ & Genotyp (G) & $* * *$ & $* * *$ \\
\hline Crop cycle (Y) & $* * *$ & $* * *$ & $\begin{array}{l}\text { Crop cycle } \\
(\mathrm{Y})\end{array}$ & $* * *$ & $* * *$ & $\begin{array}{l}\text { Crop cycle } \\
(\mathrm{Y})\end{array}$ & $* * *$ & $* * *$ \\
\hline G x Y & * & $*$ & Gx Y & $* * *$ & $* * *$ & $\mathrm{G} \times \mathrm{Y}$ & $* * *$ & $* * *$ \\
\hline Mean & 38.5 & 0.4 & Mean & 40.7 & 0.5 & Mean & 53.3 & 0.7 \\
\hline CV (\%) & 90.0 & 84.0 & CV $(\%)$ & 94.3 & 101.1 & CV $(\%)$ & 78.5 & 86.0 \\
\hline $\mathrm{SD}$ & 34.6 & 0.3 & SD & 38.4 & 0.5 & SD & 41.8 & 0.6 \\
\hline
\end{tabular}




\subsection{Cane Genotypes Resistance or Susceptibility to SCSM Disease at Three Months}

\subsubsection{Plant Cane}

Higher percentage of disease resistant genotypes were observed in V4-43 and P3-61 experiments conducted in Ferké 2 with 72 and 52\%, respectively (Fig. 2). The lowest percentage of resistant genotypes was observed in B313 experiment conducted in Ferké 1, with 37\%. Highly susceptible genotypes were observed in B3-13 and V8-01 experiments carried out in Ferké 1 and Ferké 2, respectively, with 16 and 9\% of genotypes tested.

Among all genotypes tested ( $\mathrm{N}=119), 50.4 \%$ were resistant, $22.7 \%$ moderately resistant, $22.7 \%$ susceptible and $4.2 \%$ highly susceptible (Fig. 3). About $5 \%$ of genotypes were asymptomatic and therefore supposed to be highly resistant, namely RCI12/1116, RCI13/145, RCI14/157, RCI12/192, RCI13/187 and RCI14/159 (Table 3). Their agronomic performances will be carefully checked across the selection process, as resistance to SCSMV became recently a top ranking criterion followed by sugar yield in Ferké sugar estates agro-ecology. Five genotypes, namely RCI13/172, RCI13/182, RCI13/178 RCI13/1135 and RCI13/1133, found highly susceptible alongside with susceptible and moderately resistant ones will therefore be eliminated in first ratoon after harvest for the advanced selection stage irrespective of their sugar yield performances and other agro-morphological traits like erect and self-defoliating stalks for easy mechanized green harvesting.

Symptom observations in this study were made at three or four months, therefore at early grand growth stage of sugarcane where infection could be recognized visually without any virus detection equipment like ELISA chain (Enzyme Linked Immuno Sorbent Assay) or RT-PCR (Reverse Transcription - Polymerase Chain Reaction). However, observations made later at that growth stage (5-9 months) and the formative growth stage (10-12 months) showed much higher values of disease severity ranging from 2 to $3(-)$ on susceptible genotypes compared with 0.1 to 0.5 (-) determined at early grand growth stage. That is why our suggestion would be to observe at 5-7 months, where sugarcane fields are still easily accessible for growth measurements and disease control.

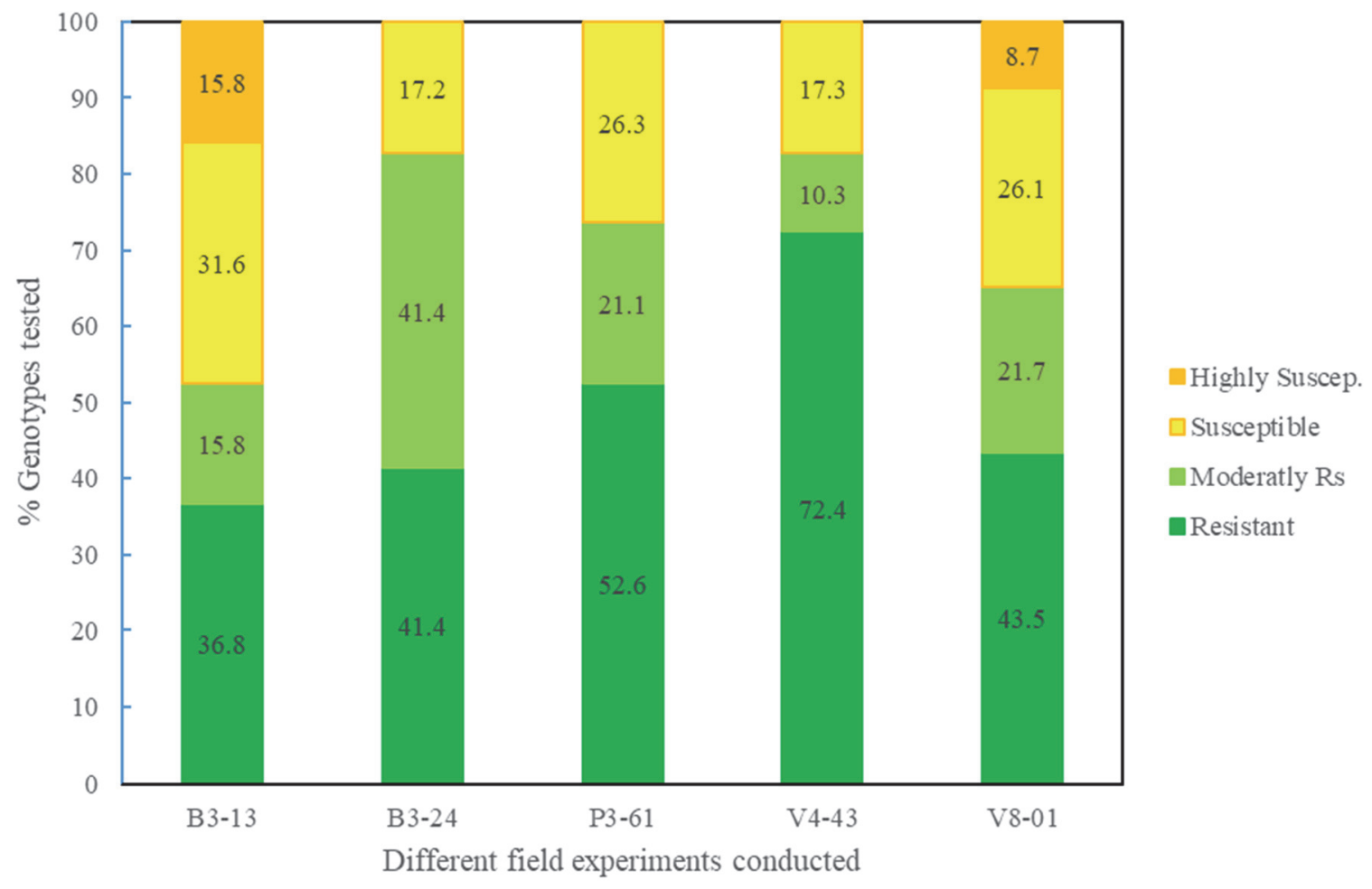

Figure 2. Sugarcane genotype resistance or susceptibility to SCSM disease in plant cane, at 3-4 months across field experiments carried out in plant crop at Ferké Sugar estates, Ivory Coast 


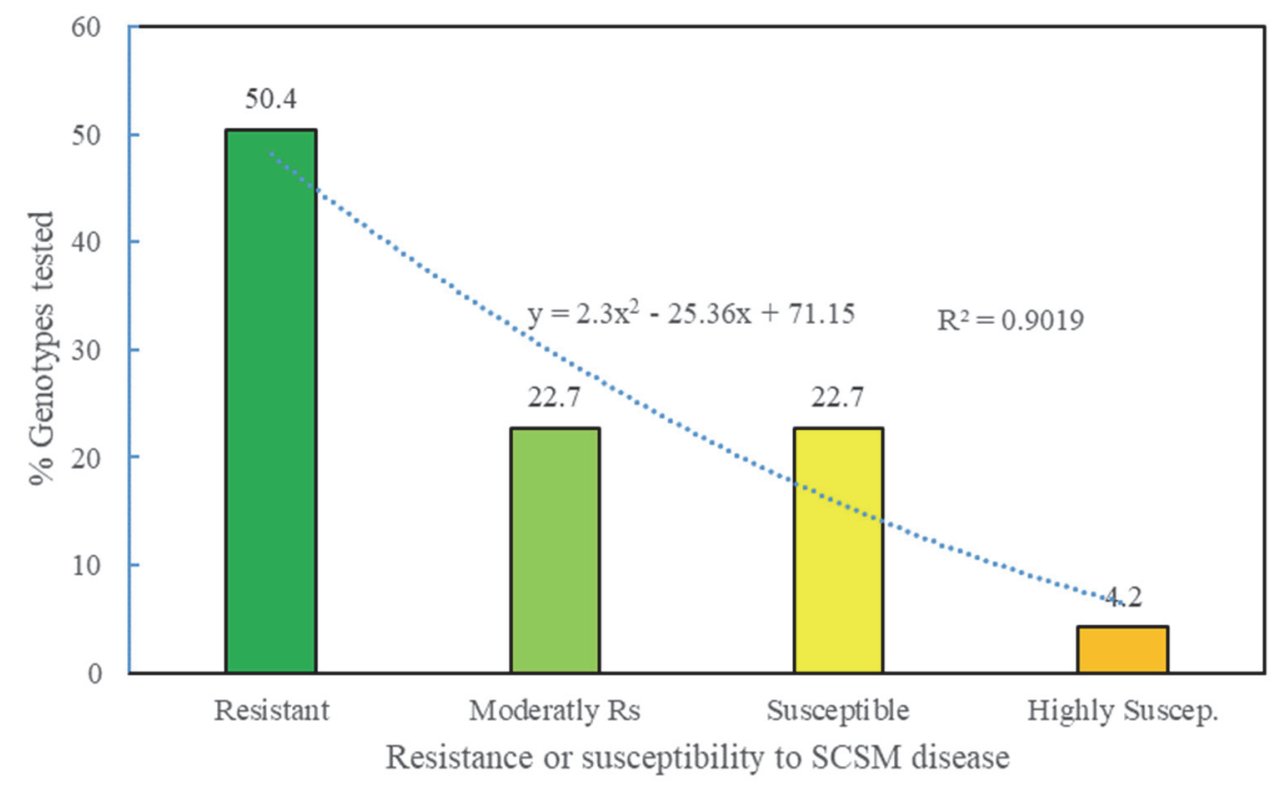

Figure 3. Percentage of genotype resistant or susceptible to SCSM disease in plant cane at early selection stage at Ferké sugar estates, Ivory Coast

Table 3. Resistant and highly susceptible cane genotypes to natural infection of SCSM disease in plant cane at Ferké sugar estates, Ivory Coast

\begin{tabular}{|c|c|c|c|c|c|}
\hline \multirow{2}{*}{ Field experiments } & \multicolumn{5}{|c|}{ Cane genotypes } \\
\hline & \multicolumn{3}{|c|}{ Resistant } & \multicolumn{2}{|l|}{ Highly susceptible } \\
\hline & RCI11/170; & RCI14/1 & & RCI13/172 ; & RCI13/182; \\
\hline \multirow{4}{*}{ B3-13/Ferké 1} & RCI13/183; & RCI13/1 & & RCI13/178 & \\
\hline & RCI11/163; & RCI14/16 & & & \\
\hline & RCI10/164 & & & & \\
\hline & RCI12/1116* & RCI13/112 & & & \\
\hline \multirow[t]{6}{*}{ B3-24/Ferké 1} & RCI14/1109; & RCI14/1105 & & - & \\
\hline & RCI14/1101 ; & RCI13/1123 & & & \\
\hline & RCI13/1124; & $\mathrm{RCI13/111S}$ & & & \\
\hline & RCI13/197; & RCI14/1108 & & & \\
\hline & RCI13/1117; & RCI14/1107 & & & \\
\hline & RCI13/145*; & RCI14/157* & & & \\
\hline \multirow[t]{5}{*}{ P3-61/Ferké 2} & RCI14/147; & RCI13/15? & & - & \\
\hline & RCI13/151 ; & RCI14/15 & & & \\
\hline & RCI13/144 ; & RCI14/15t & & & \\
\hline & RCI13/138; R & RCI13/142 & & & \\
\hline & RCI12/192* & ; RCI13/187* & ; & & \\
\hline \multirow[t]{11}{*}{ V4-43/Ferké 2} & RCI14/159*; & RCI14/128 & ; & & \\
\hline & RCI13/13 & ; $\quad$ RCI13/194 & ; & - & \\
\hline & RCI13/139 & RCI13/173 & ; & & \\
\hline & RCI11/135 & RCI13/16 & ; & & \\
\hline & RCI14/188 & RCI13/110 & ; & & \\
\hline & RCI13/193 & RCI11/162 & ; & & \\
\hline & RCI11/112 & RCI12/191 & ; & & \\
\hline & RCI13/195 & RCI14/189 & ; & & \\
\hline & RCI14/111 & RCI11/134 & ; & & \\
\hline & RCI13/196 & & & & \\
\hline & RCI13/1136; & RCI13/1132 & & & \\
\hline
\end{tabular}




\begin{tabular}{|c|c|c|c|}
\hline V8-01/Ferké 2 & $\begin{array}{l}\text { RCI14/1140; } \\
\text { RCI14/1139; } \\
\text { RCI14/1138; } \\
\text { RCI14/1145; }\end{array}$ & $\begin{array}{l}\text { RCI14/1141; } \\
\text { RCI14/1142; } \\
\text { RCI14/1137; } \\
/ 1147\end{array}$ & RCI13/1135 ; RCI13/1133 \\
\hline
\end{tabular}

*: asymptomatic genotypes $(\mathrm{n}=6)$

\subsubsection{First Ratoon Crop}

In the first ratoon crop, higher percentage of highly susceptible genotypes to SCSM disease (86-95.7\%) were observed in all experiments conducted in both sugar estates (Fig. 3). Percentage of resistant genotypes observed in Ferké 2 ranged from 4.3 to $6.9 \%$ while none of them was observed in both Ferké 1 trials. That of susceptible genotypes ranged from 5.3 to $6.9 \%$ observed in both Ferké 1 trials as well as V4-43 trail of Ferké 2 . In total, about $92 \%$ of genotypes tested were highly susceptible whereas 4.2 and $3.4 \%$ were susceptible and resistant, respectively (Fig 4, table 4).

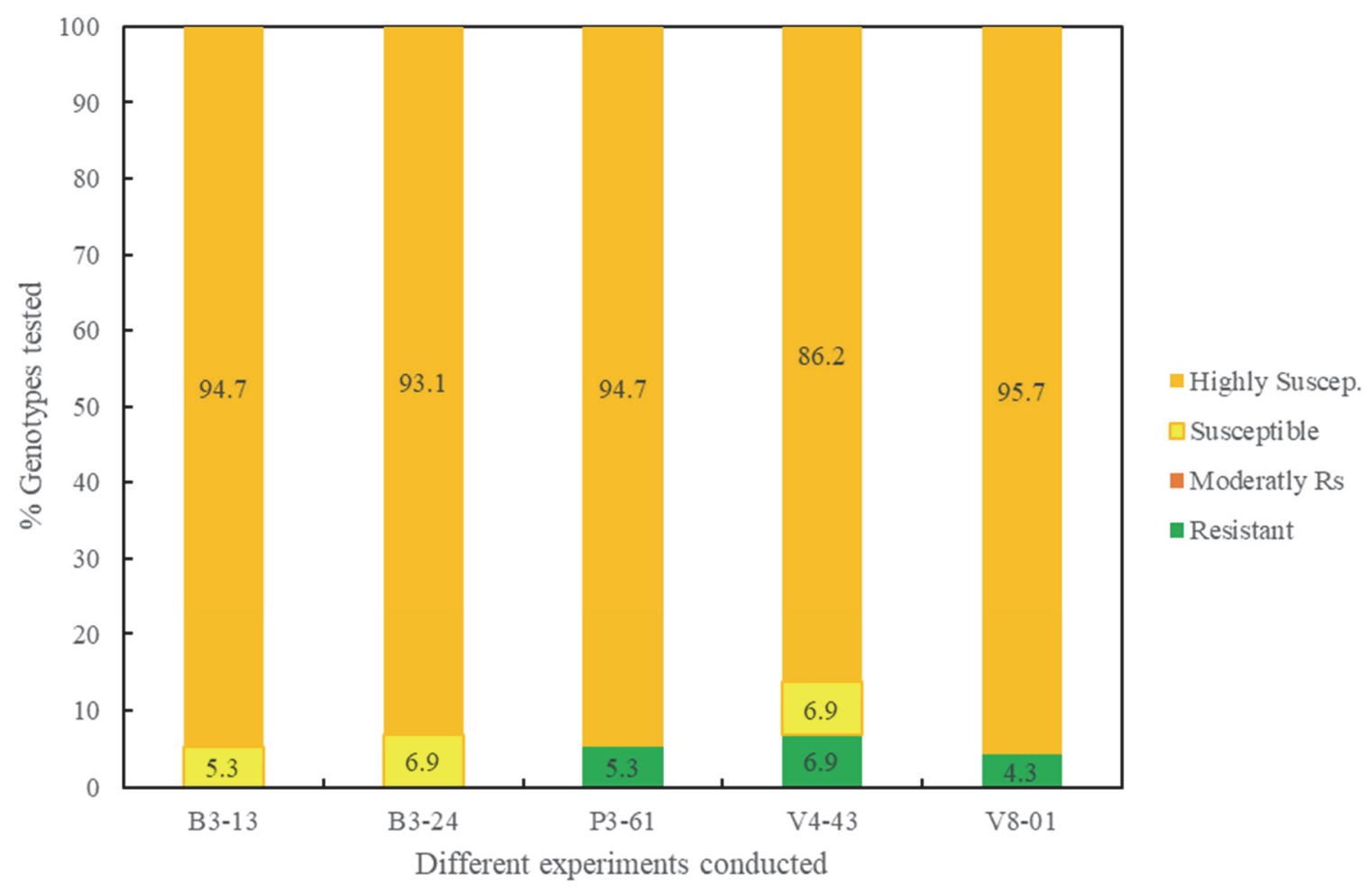

Figure 3. Sugarcane genotype resistance or susceptibility to SCSM disease in first ratoon crop at 3-4 months, across experiments carried out in plant crop at Ferké Sugar estates, Ivory Coast 


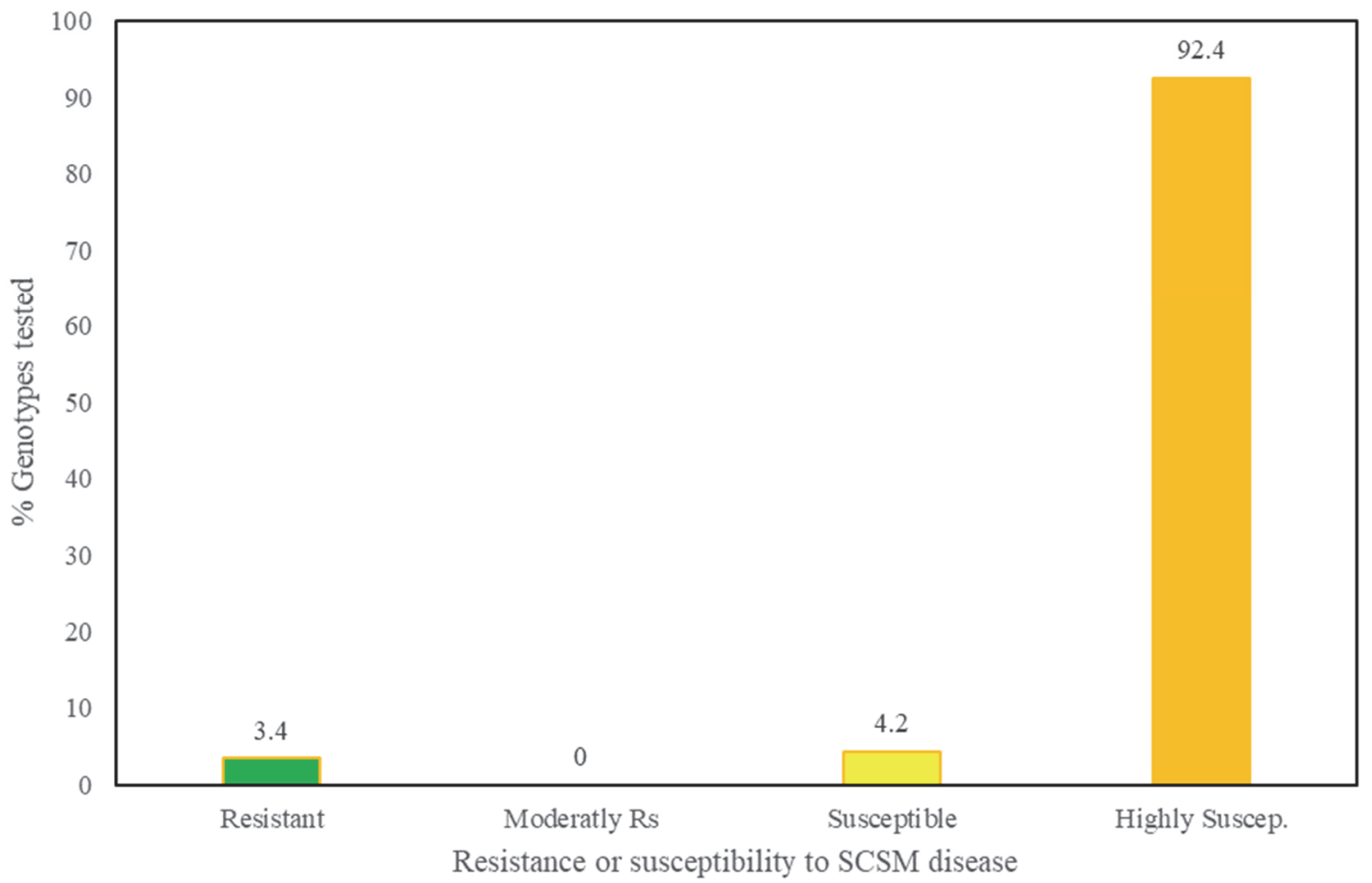

Figure 4. Percentage of genotype resistant or susceptible to SCSM disease in first ratonn crop at early selection stage at Ferké sugar estates, Ivory Coast

Table 4. Resistant and highly susceptible cane genotypes to natural infection of SCSM disease in first ratoon crop at Ferké sugar estates, Ivory Coast

\begin{tabular}{|c|c|c|c|c|c|}
\hline & \multicolumn{5}{|l|}{ Cane genotypes } \\
\hline \multirow[t]{2}{*}{ Field experiments } & Resistant & Highly susce & $\mathrm{ti}$ & & \\
\hline & & RCI10/164 & - & RCI11/163 & - \\
\hline \multirow[t]{10}{*}{ B3-13/Ferké 1} & - & RCI11/167 & - & RCI11/168 & - \\
\hline & & RCI11/169 & - & RCI11/170 & - \\
\hline & & RCI13/172 & - & RCI13/175 & - \\
\hline & & RCI13/176 & - & RCI13/178 & - \\
\hline & & RCI13/181 & - & RCI13/182 & - \\
\hline & & RCI13/184 & - & RCI13/185 & - \\
\hline & & RCI13/186 & - & RCI14/158 & - \\
\hline & & RCI14/160 & & & - \\
\hline & & RCI14/161 & & & \\
\hline & & RCI13/1121 & . & RCI13/1122 & - \\
\hline \multirow[t]{11}{*}{ B3-24/Ferké 1} & - & RCI13/1117 & . & RCI13/1124 & - \\
\hline & & RCI12/1116 & & RCI11/1110 & - \\
\hline & & RCI11/1112 & & $\mathrm{RCI} 13 / 1120$ & - \\
\hline & & RCI14/199 & & RCI13/1119 & - \\
\hline & & RCI11/1111 & & RCI13/1123 & - \\
\hline & & RCI14/1108 & & RCI14/1109 & - \\
\hline & & RCI11/1113 & & RCI14/1101 & - \\
\hline & & RCI14/1107 & & RCI11/1114 & - \\
\hline & & RCI13/198 & & RCI14/1102 & - \\
\hline & & RCI14/1100 & & RCI14/1104 & - \\
\hline & & RCI13/1118 & . & RCI14/1103 & - \\
\hline
\end{tabular}


P3-61/Ferké 2

V4-43/Ferké 2

V8-01/Ferké 2
RCI13/145

RCI13/16; RCI13/187

RCI13/1132

\begin{tabular}{|c|c|c|c|}
\hline \multirow{2}{*}{$\begin{array}{l}\text { RCI14/1105 } \\
\text { RCI14/1125 }\end{array}$} & - & RCI14/1106 & - \\
\hline & & & \\
\hline RCI13/150 & - & RCI13/153 & - \\
\hline RCI13/142 & - & RCI14/157 & - \\
\hline RCI12/149 & - & RCI14/146 & - \\
\hline RCI13/138 & - & RCI13/144 & - \\
\hline RCI14/154 & - & RCI13/151 & - \\
\hline RCI13/143 & - & RCI13/152 & - \\
\hline RCI14/155 & - & RCI14/148 & - \\
\hline RCI13/140 & - & RCI14/156 & - \\
\hline RCI14/147 & & & - \\
\hline RCI13/141 & & & \\
\hline RCI11/135 & - & RCI14/159 & - \\
\hline RCI11/112 & - & RCI13/110 & - \\
\hline RCI13/139 & - & RCI14/111 & - \\
\hline RCI13/193 & - & RCI11/134 & - \\
\hline RCI14/128 & - & RCI13/13 & - \\
\hline RCI13/196 & - & RCI11/162 & - \\
\hline RCI12/191 & - & RCI11/190 & - \\
\hline RCI13/195 & - & RCI11/165 & - \\
\hline RCI13/180 & - & RCI14/188 & - \\
\hline RCI14/189 & - & RCI13/177 & - \\
\hline RCI13/174 & - & RCI11/166 & - \\
\hline RCI14/171 & - & RCI13/173 & - \\
\hline RCI13/179 & & & \\
\hline RCI14/1145 & - & RCI13/1136 & - \\
\hline RCI13/1134 & - & RCI14/1139 & - \\
\hline RCI14/1141 & - & RCI14/1144 & - \\
\hline RCI13/1133 & - & RCI14/1140 & - \\
\hline RCI11/1128 & - & RCI14/1147 & - \\
\hline RCI11/1129 & - & RCI14/1142 & - \\
\hline RCI14/1143 & - & RCI14/1137 & - \\
\hline RCI14/1126 & - & RCI11/1146 & - \\
\hline RCI12/1130 & - & RCI13/1131 & - \\
\hline RCI13/1135 & - & RCI13/1148 & - \\
\hline RCI14/1127 & & & - \\
\hline RCI14/1138 & & & \\
\hline
\end{tabular}

\subsection{Disease Epidemiology}

Some weed species like Rottboellia exaltata L, Dactyloctenium aegyptium, Imperata cilyndrica, Pennisetum pupureum, Paspalum spp., Bracharia moliniformis and Panucum repens, as well as cereal crops like maize (Zea mays L) and sorghum (Sorghum bicolor) are respectively highly endemic and commonly cultivated across Ivorian sugar estates. Several investigators reported that they were host plants for SCSMV (Putra et al, 2014; Prabowo et al, 2014).

Although investigations were made on suspected insect vectors of the disease like Ceratovacum lanigera Zeh., Rhopalosiphum maydis, Saccharicocus sacchari Cock., and Melanaphis sacchari Zeh. (a sugarcane aphid) (Damayanti \& Putra, 2011; Putra \& Damayanti, 2012; Béhou \& Péné, 2018), no evidence of disease transmission from a vector was made to our knowledge.

Under Ivorian field conditions, two insect species (Locris rubra and L. maculate) were suspected as possible SCSMV vectors. They were frequently observed on susceptible varieties namely R579, SP81-3250, and R570. Beside them, numerous possible vectors used to be observed on leaves of susceptible varieties such as Zonecerus variegatus, Paracinema tricolor, Stenohippus aequus, Oxya hyla and Conocephalus longipennis. In addition to the fact that SCSM disease is spread very fast, similarly to the case of rice yellow mottle virus, it could be hypothesized that a complex biocenosis of pests could be possible vectors of the disease (Koudamiloro et al, 2015). 
SCSMV constitutes a great challenge for geneticists, breeders, pathologists, agronomists as well as cane growers. In the sense that it is not eliminated by hot water treatment (HWT), in contrast to most economically important diseases in sugarcane frequent in sub-Saharan Africa and elsewhere like leaf scald (Xanthomonas albinineans), smut (Sporisorium scitamineum), yellow leaf disease (SCYLD), orange rust (Puccinia kuehnii), RSD (Leifsonia $x y l i$ subsp. Xyli), and brown rust (Puccinia melanocephala). Other challenges lie in the fact that no insect vector of SCSMV is formally identified yet, its transmission mechanism from vector to crop being still unknown and it spreads very fast across sugarcane fields and varieties, with significant yield reduction as reported in Brazil, Indonesia and Ivory Coast (Gonçalves et al, 2007b; Magarey et al, 2018; Béhou \& Péné, 2019. Prophylactic measures based on agricultural practices regarding sanitation of planting and harvesting tools or machinery as well as search for resistant cane varieties must be given a top priority. In this regard, sugarcane selection starting from true seed constitutes a great opportunity to broaden the genetic diversity of plant material being tested in line of SCSMV thread. This program is being under way since 2014-15 in Ivory Coast, and much earlier in Senegal and some Central African countries (Cameroon, Tchad and Congo), in collaboration with R\&D institutes like eRcane (Reunion Island) and MSIRI (Mauritius).

\section{Conclusions}

In each of the five selection trails conducted on both Ferké $1 \& 2$ sugar estates, highly significant differences in disease incidence and severity were observed between genotypes as well as crop cycles (plant cane and first ratoon). Except for one trial (B3-24 in Ferké 1), Genotype x crop cycle interactions were significant or highly significant, which showed that the majority of genotypes tested behaved differently from plant cane to first ratoon towards the disease. Particularly, the percentage of resistant genotypes decreased from 50 to $3.4 \%$ whereas that of highly susceptible ones increased from 4.2 to $92.4 \%$. This shows the level of challenge to be tackled in the control of SCSM disease through sugarcane breeding and selection. At the end of the current selection stage under way, i.e. after harvest of first ratoon, only the best yielding genotypes among the resistant ones will undergo the advanced selection stage.

\section{References}

Addy, H. S., Nurmalasari, Wahyudi, A. H. S., Sholey, A., Anugrah, C., Iriyanto, F. E. S., Darmanto W., \& Sugiharto, B. (2017). Detection and response of sugarcane against the infection of Sugarcane Mosaic Virus (SCMV) in Indonesia. Agron., 7(50), 1-11. https://doi.org/10.3390/agronomy7030050

Béhou, Y. M., \& Péné C. B. (2018). Genetic variability of sugarcane clones as affected by endemic diseases at onle-row screening stage in Ferké, Northern Ivory Coast. $J$ Experim. Agric. Int., 24(5), 1-14. https://doi.org/10.9734/JEAI/2018/42266

Béhou, Y. M., \& Péné, C. B. (2019). Genetic variability of sugarcane clones as affected by major endemic diseases in Ferké, Northern Ivory Coast. Advances and Trends in Agric. Sci., 1, 65-80. https://doi.org/10.9734/JEAI/2018/42266

Brown, J. (1997). Survival and dispersal of plant parasites: general concepts. In Brown, J. F., \& Ogle, H. J. (Eds.), Plant Pathogens and Plant Diseases (pp. 195-206). Rockvale Publications, Amidale. NSW-Australia.

Chatenet, M., Mazarin, C., Girad, J. C., Gargani, D., Rao, G. P., Royer, M., Lockhart, B. E. L., \& Rott, P. (2005). Detection of sugarcane streak mosaic virus in sugarcane from several Asian countries. Sugar Cane Int., 23(4), $12-5$.

Damayanti, T. A., \& Putra, L. K. (2011). First occurrence of sugarcane streak mosaic virus infecting sugarcane in Indonesia. J. General Plant Pathol, 7(1), 72-4. https://doi.org/10.1007/s10327-010-0285-7

Gomez, K. A., \& Gomez, A. A. (1984). Statistical procedure for agricultural research (2nd ed). John Wiley and Sons Inc, New York.

Gonçalves, M. C., Galdeano, D. M., Maia, I. G., \& Chagas, C. M. (2011). Genetic variability of SCMV genotypes causing maize mosaic in Brazil. Agric. Res., 46, 362-9. https://doi.org/10.1590/S0100-204X2011000400004

Gonçalves, M. C., Maia, I. G., Galleti, S. R., \& Faustin, G. M. (2007b). Mixed infection by SCMV and MSV causing breaking yields in maize in Sao Paulo State. Summa Phytopathol, 33, 22-6. https://doi.org/10.1590/S0100-54052007000400005

Gonçalves, M. C., Moreira, Y. J. C. B., Maia, I. G., Santos, A. S., Faustin, G. M., \& Chaves, A. (2004). Identification and characterization of solates from SCMV sub-group in San Paulo state, Brazil. Phytopathol. Brasileira, 32, 32-9.

Gonçalves, M. C., Pinto, L. R., Souza, S. C., \& Landell, M. G. A. (2012). Virus diseases of sugarcane. A constant 
challenge to sugarcane breeding in Brazil. Func. Plant Sci. Biotech, 6(special issue 2), 108-16.

Gonçalves, M. C., Santos, A. S., Maia, I. G., Chagas, C. M., \& Harakava, R. (2007a). Characterization of an isolate of sugarcane mosaic virus breaking down resistance of commercial sugarcane varieties. Phytopathol. Brasileira 29: 129-39.

Hema, M., Savithri, H. S., \& Sreenivasulu, P. (2001). Sugarcane streak mosaic virus: occurrence, purification, characterization and detection. In Rao, G. P., Ford, R. E., Tosic, M., \& Teakle, D. S. (Eds.), Sugarcane Pathol. Vol II: Viruses and Phytoplasma Diseases. Science Publishers Inc. Enfield USA: 37-70.

Konan, E. A., Péné, C. B., \& Dick, E. (2017a). Main factors determining the yield of sugarcane plantations on Ferralsols in Ferké 2 sugar complex, Northern Ivory Coast. J. Emerg. Trends Engineer. Appl. Sci. JETEAS, 8(6), 244-256.

Konan, E. A., Péné, C. B., \& Dick, E. (2017b). Caractérisation agro-climatique du périmètre sucrier de Ferké 2 au Nord de la Côte d'Ivoire. J Appl. Biosci., 116, 11532-11545. https://doi.org/10.4314/jab.v116i1.2

Koudamiloro, A., Nwilene, F. E., Togola, A., \& Akogbeto, M. (2015). Insect vectors of rice yellow mottle virus. $J$. Insects (ID721751, 1-12. https://doi.org/10.1155/2015/721751

Kristini, A. I., \& Sasongko, D. (2006). Mosaic booming. Gula Indonesia, 30(1), 36-38 (in Indonesian).

Li, W., He, Z., Li, S., Huang, Y., Zhang, Z., Jiang, D., Wang, X., \& Luo, Z. (2011). Molecular characterization of a new strain of sugarcane streak mosaic virus (SCSMV). Arc. Virol., 156, 2101-04. https://doi.org/10.1007/s00705-011-1090-0

Magarey. R. C., Kristini, A., Achadian, E., Thompson, N., Wilson, E., Reynolds, M., Sallam, N. R., Goebel, F. R., \& Putra, L. (2018). Sugarcane streak mosaic: researching a relatively new disease in Indonesia. Proc. Aust. Soc. Sugar Cane Technol, 40, 257-66.

Prabowo, D. B., Hadiastono, T., Himawan, T., \& Putra, L. K. (2014). Detection disease of Sugarcane Streak Mosaic Virus (SCSMV) via serological test on sugarcane (Saccharum officinarum L.) weed and insect vector. Int. J. Sci. Res. (IJSR), 3(1), 88-92.

Putra, L. K, Astono, T. H., Syamsidi, S. R. C., \& Djauhari, S. (2015a). Dispersal, yield losses and varietal resistance to sugarcane streak mosaic virus (SCSMV) in Indonesia. Intern. $J$ Virol., 11(1), 32-40. https://doi.org/10.3923/ijv.2015.32.40

Putra, L. K., \& Damayanti, T. A. (2012). Major diseases affecting sugarcane production in Indonesia. Function. Plant Sci. Biotech, 6(2), 124-9.

Putra, L. K., Kristini, A., Achadian, E. M., \& Damayanti, T. A. (2014). Sugarcane streak mosaic virus in Indonesia: Distribution, characterization, yield losses and management approaches. Sugar Tech., 16(4), 392-9. https://doi.org/10.1007/s12355-013-0279-9

Shitahum, A., Feyissa, T., \& Abera, D. (2018). Performances evaluation of advanced sugarcane genotypes (Cirad 2013) at Metahara sugar estate, Ethiopia. Int. J Adv. Res. Biol. Sci., 5(1), 91-104.

Xu, D. L., Zhou, G. H., Xie, Y. J., \& Mock, R, Li. R. (2010). Complete nucleotide sequence and taxonomy of sugarcane streak mosaic virus, member of a novel genus in the family Potiviridae. Virus Gene, 40, $432-9$. https://doi.org/10.1007/s11262-010-0457-8

\section{Copyrights}

Copyright for this article is retained by the author(s), with first publication rights granted to the journal.

This is an open-access article distributed under the terms and conditions of the Creative Commons Attribution license (http://creativecommons.org/licenses/by/4.0/). 